\title{
Real-World Outcomes after 36-Month Treatment with Ranibizumab 0.5 mg in Patients with Visual Impairment due to Diabetic Macular Edema (BOREAL-DME)
}

\author{
Pascale Massin $^{\mathrm{a}}$ Catherine Creuzot-Garcher ${ }^{\mathrm{b}}$ Laurent Kodjikian ${ }^{\mathrm{c}}$ \\ Jean-François Girmens ${ }^{d}$ Cécile Delcourt ${ }^{\mathrm{e}}$ Franck Fajnkuchen ${ }^{\mathrm{f}, \mathrm{g}}$ \\ Agnès Glacet-Bernard $^{h}$ Pierre-Jean Guillausseau ${ }^{i}$ Françoise Guthux ${ }^{j}$ \\ Patrick Blink ${ }^{k}$ Angela Grelaudk \\ ${ }^{a}$ Ophthalmology, APHP Lariboisiere, Centre Breteuil, Paris, France; ${ }^{b}$ Ophthalmology, University Hospital, Dijon, \\ France; 'Ophthalmology, Hopital de la Croix Rousse, Lyon University, UMR CNRS MATEIS 5510, Lyon, France; \\ dOphthalmology, CHNO des Quinze-Vingts, Paris, France; eInserm, Bordeaux Population Health Research Center, \\ Team LEHA, UMR 1219, University of Bordeaux, Bordeaux, France; 'Ophthalmology, Hôpital Avicenne, Bobigny, \\ France; ${ }^{9}$ Centre d'Imagerie et Laser, Paris, France; hophthalmology, Centre Hospitalier Intercommunal de Créteil, \\ Paris-Est Créteil University, Créteil, France; 'Internal Medicine, Hopital Lariboisière, Paris, France; 'Novartis Pharma \\ SAS, Rueil-Malmaison, France; ${ }^{B}$ Bordeaux PharmacoEpi, INSERM CIC1401, Université de Bordeaux, Bordeaux, France
}

\section{Keywords}

Diabetic macular edema · Vascular endothelial growth factor - Ranibizumab - Best-corrected visual acuity

\begin{abstract}
Purpose: To assess the efficacy, safety, and follow-up of 36-month treatment with ranibizumab in patients with diabetic macular edema (DME) in real-life setting. Methods: This is a prospective phase 4 observational study. Between December 2013 and April 2015, 84 ophthalmologists enrolled a total of 290 adult patients initiating ranibizumab for visual impairment due to DME and treated them according to their routine practice. The primary outcome (mean change in best-corrected visual acuity [BCVA] after 12 months) was previously reported. Here, we present outcomes after 36 months of follow-up for BCVA and change in central subfield thickness (CSFT) and report how participating ophthalmolo-
\end{abstract}

karger@karger.com

(c) 2020 S. Karger AG, Basel

www.karger.com/ore

Karger" gists treated DME over a 3-year period (number of visits and injections and evolution of treatment strategy). Results: Of the 290 patients enrolled, 187 (64.5\%) completed the 36 months of the study (entire cohort). In the entire cohort, 97 patients were treated exclusively with ranibizumab throughout the study, and 90 patients switched to other intravitreal treatments. Mean BCVA was 64.2 (20.1) letters, representing a gain of +4.1 (19.9) letters from baseline to month 36 (M36). CSFT improved over the study, and by M36 had decreased by 127 (138) $\mu \mathrm{m}$ compared to baseline. Over the 36 months of follow-up, patients in the entire cohort paid their ophthalmologists a mean of 30.9 (12.2) visits and had a mean of 7.6 (5.2) any injections. Results for quality of life questionnaires NEI-VFQ25 and HUI-3 remained stable throughout the study. Multivariate analysis on the 145 patients with evaluable BCVA data at M36 found that male gender and milder baseline DME characteristics (BCVA $\geq 59$ and CSFT $<500 \mu \mathrm{m}$ ) were predictive factors for achieving a BCVA of $\geq 70$ letters at M36. 
This study did not find any new safety signals, compared to the known profile of ranibizumab. Conclusions: Gains in BCVA in this real-life study were lower than those observed in randomized clinical trials with ranibizumab, mainly due to undertreatment. Safety analysis of ranibizumab did not yield any new safety concerns.

(c) 2020 S. Karger AG, Basel

\section{Introduction}

Diabetic macular edema (DME) secondary to diabetic retinopathy is a leading cause of visual impairment in people with type 1 and type 2 diabetes $[1,2]$. DME is estimated to affect 21 million people worldwide [3].

The mechanisms underlying the pathogenesis of DME have not been completely elucidated, but it is known that hyperglycemia leads to accumulation of fluid in the retina (edema) via increased vascular permeability of the blood retinal barrier. Vascular endothelial growth factor (VEGF) has been implicated in regulating retinal vascular permeability.

Ranibizumab (Lucentis ${ }^{\circledR}$; Novartis) is a Fab fragment of a humanized monoclonal antibody that acts as a highaffinity VEGF antagonist. Ranibizumab $0.5 \mathrm{mg}$, administered by intravitreal injection, received initial EMA approval in 2007 for age-related macular degeneration. The indication was extended in 2011 to DME based on improved visual acuity and anatomic parameters demonstrated in the phases 2 and 3 randomized controlled trials (RCTs) RESOLVE and RESTORE [4-6]. Ranibizumab $0.3 \mathrm{mg}$ was approved for DME by the FDA in 2012 based on the RISE and RIDE trials. These were identical parallel phase 3 RCTs in the USA comparing 2 years of treatment with ranibizumab monotherapy ( 0.3 and $0.5 \mathrm{mg}$ ) to laser monotherapy, with a 1-year open-label extension [7].

The RCTs underpinning the marketing authorization of ranibizumab tested efficacy and safety in tightly controlled conditions, in selected populations. These conditions do not always represent practices in real life. Therefore, postauthorization studies are useful to learn how medicines are prescribed and used in real-world settings.

This study was mandated by the French Transparency Commission during the approval of ranibizumab reimbursement in DME, with the aim of providing data on the efficacy, safety, and follow-up in a real-life setting in DME patients initiating treatment with ranibizumab. The year 1 results of this study demonstrated the efficacy and safety of ranibizumab treatment, and no new ocular or non- ocular safety concerns were observed [8]. Here, we describe the results of a 3-year postauthorization study of ranibizumab in DME.

\section{Materials and Methods}

Study Design and Patients

This was a mandatory phase 4 prospective 36-month noninterventional postauthorization cohort study conducted in France between December 2013 and April 2015. French retinal expert ophthalmologists (both community and hospital based) included on a national listing held by the company CEGEDIM were invited to become investigators.

Eligible patients were adults with type 1 or 2 diabetes who started ranibizumab for visual impairment due to DME. For patients with bilateral DME at inclusion, 1 eye was randomly selected to be the studied eye. Each participating ophthalmologist could recruit up to 30 consecutive eligible and consenting patients over a period of 16 months.

All patients signed an informed consent form. The study protocol was approved by the relevant committees in France for data protection (CNIL) and for managing health research data (CCTIRS). In line with French regulations for observational studies, ethical approval was not required.

\section{Treatment}

Patients were treated with intravitreal ranibizumab according to the ophthalmologist's routine practice. Investigating ophthalmologists retreated patients at their discretion, following treat and extend (T\&E) or pro re nata (PRN, "as needed") regimens as appropriate.

\section{Data}

Data were obtained from the medical records of participants by investigators or their delegates at inclusion and at every medical visit (usually every 3 months) up to month 36 (M36). The variables of interest were described previously [8]. Briefly, the data recorded at the inclusion visit were demographics, diabetes and ocular disease characteristics, comorbidities, treatment details, and adverse events (AEs). At each subsequent visit, data were recorded for treatments (including visits, DME and other ocular treatments, and reasons for discontinuation, retreatment, or switching), visual acuity (ETDRS letters, converted from Monoyer or Snellen charts where necessary), central subfield retinal thickness (CSFT), and safety (AEs and glycemic control).

In addition, patients were telephoned at baseline and every 6 months to complete the QoL questionnaires National Eye Institute Visual Function Questionnaire (NEI-VFQ) and Health Utilities Index-3 (HUI-3). NEI-VFQ responses were used to calculate the global score of visual function, as well as scores for general vision, ocular pain, near vision activities, far vision activities, social life, mental health, dependence, driving, color vision, and peripheral vision. The HUI-3 global score assesses the level of disability on a scale of 1 (no disability) to 4 (severe disability).

\section{Sample Size and Data Analysis}

The sample size calculation and statistical principles were described previously [8]. This article presents data from the full 36-month study duration. 


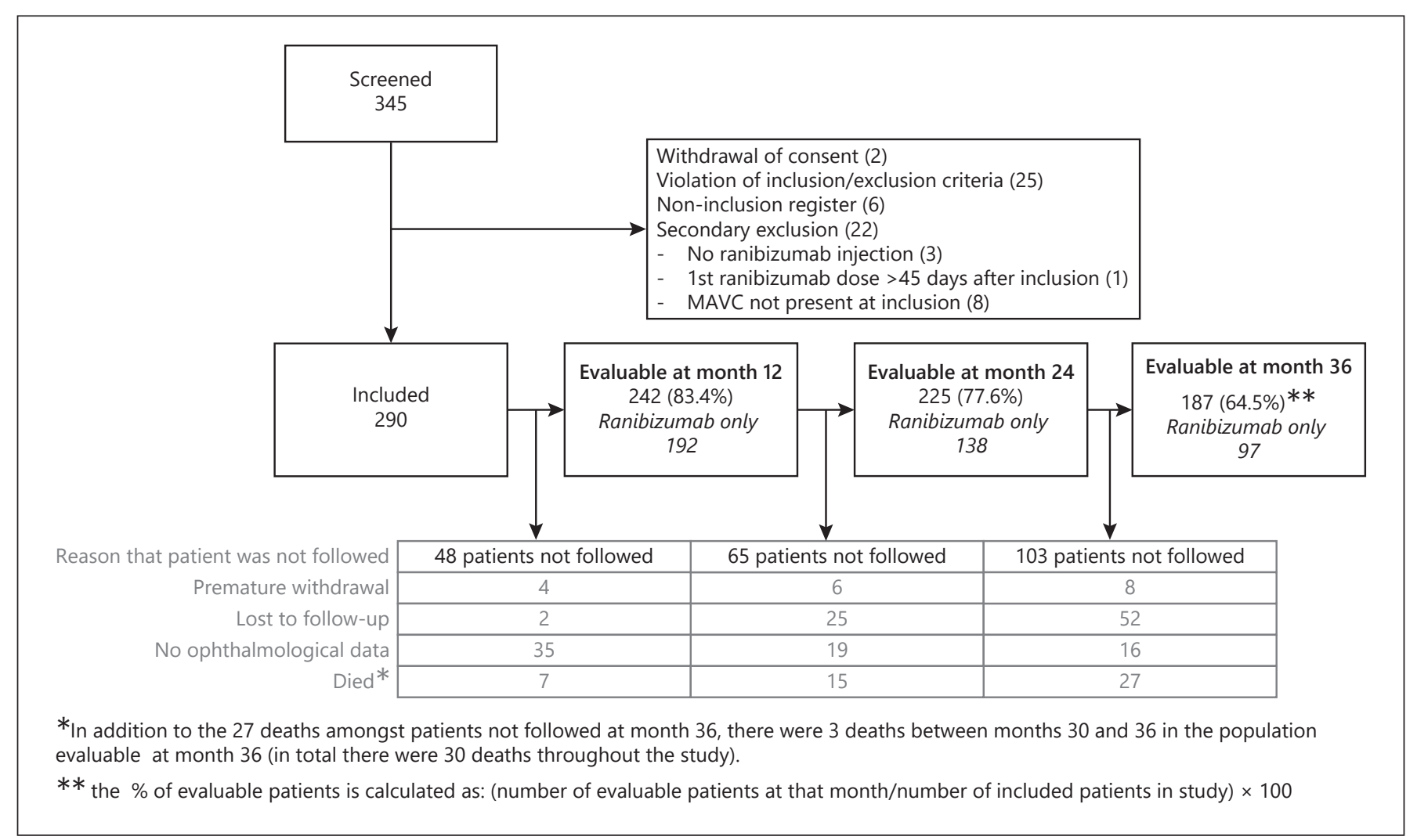

Fig. 1. Flow chart tracking the number of patients evaluable at each time point (one studied eye per patient).

Results for the primary endpoint, change in best-corrected visual acuity (BCVA) from baseline to month 12, were previously reported [8]. Secondary endpoints were BCVA and CSFT change from baseline to M36, safety, characteristics of ranibizumab treatment (frequency of visits and injections and switch to other treatments), and quality of life assessment.

A complementary analysis for all endpoints was performed for the "ranibizumab-only subgroup" of patients whose only intravitreal treatment up to M36 was ranibizumab (i.e., they did not receive intravitreal injection of dexamethasone, triamcinolone, bevacizumab, pegaptanib, or aflibercept). Missing data were not replaced for the core analyses presented in this study. Sensitivity analyses were performed for the BCVA up to M36 with missing data replaced by last observation carried forwards (LOCF) and using a Markov chain Monte Carlo algorithm for multiple imputation.

\section{Results}

\section{Patients}

Of the 1,666 ophthalmologists (both hospital and community based) invited to participate as investigators,
84 agreed and included a total of 290 patients. Of these patients, $187(64.5 \%)$ completed the 36 months of the study, of whom 97 patients (51.9\%) were treated exclusively with ranibizumab and no other intravitreal medication. The remaining patients withdrew, were lost to follow-up (despite efforts to contact), did not have ophthalmological data, or died (see Fig. 1). The group of patients not followed at M36 (103 patients) had several differences compared to the group of patients followed at M36. They were slightly older (mean age was 68.0 [12.7] years vs. 65.1 [9.5] years), and they had higher rates of cardiovascular events (31.1 vs. $24.6 \%$ ) and stroke (9.7 vs. $6.4 \%$ ). Patients not followed at M36 had more unilateral DME (50.5 vs. $26.7 \%$ ) and less bilateral DME (48.5 vs. $72.7 \%$ ). They also had a lower rate of previous treatments in the studied eye for reduced VA due to DME (9.7 vs. 14.4\%).

Just over half $(53.5 \%)$ of participants who completed the study were male, and the mean (SD) age overall was 65.1 (9.5) years (Table 1). Most patients had type 2 diabetes $(83.4 \%)$. The mean time from diabetes diagnosis to inclusion was 17.7 (11.5) years. The mean time from di- 
Table 1. Baseline demographics and disease characteristics for patients (or studied eyes) followed at month 36

\begin{tabular}{|c|c|c|}
\hline & $\begin{array}{l}\text { Entire cohort, } \\
n=187\end{array}$ & $\begin{array}{l}\text { Ranibizumab-only } \\
\text { subgroup, } n=97\end{array}$ \\
\hline \multicolumn{3}{|l|}{ Sex, $n(\%)$} \\
\hline Male & $100(53.5)$ & $52(53.6)$ \\
\hline Female & $87(46.5)$ & $45(46.4)$ \\
\hline Age, years, mean (SD) & $65.1(9.5)$ & $65.1(9.5)$ \\
\hline \multicolumn{3}{|l|}{ Diabetes } \\
\hline \multicolumn{3}{|l|}{ Type of diabetes, $n(\%)$} \\
\hline Type I & $31(16.6)$ & $20(20.6)$ \\
\hline Type II & $156(83.4)$ & $77(79.4)$ \\
\hline Time since diagnosis of diabetes, years, mean (SD) & $17.7(11.5)$ & $16.9(10.6)$ \\
\hline $\mathrm{BMI}, \mathrm{kg} / \mathrm{m}^{2}$, mean $(\mathrm{SD})$ & $30.2(6.0)$ & $30.3(5.5)$ \\
\hline \multicolumn{3}{|l|}{ Diabetic retinopathy in studied eye, $n(\%)$} \\
\hline Nonproliferative & $126(67.4)$ & $65(67.0)$ \\
\hline Proliferative & $22(11.8)$ & $10(10.3)$ \\
\hline Inactivated by PRP & $37(19.8)$ & $20(20.6)$ \\
\hline \multicolumn{3}{|l|}{ DME } \\
\hline Bilateral DME, $n(\%)$ & $136(72.7)$ & $64(66.0)$ \\
\hline \multicolumn{3}{|c|}{ Time since diagnosis of DME in the studied eye, categories, $n(\%)$} \\
\hline$<1$ month & $55(29.4)$ & $32(33.0)$ \\
\hline $1-2$ months & $14(7.5)$ & $9(9.3)$ \\
\hline $3-6$ months & $24(12.8)$ & $9(9.3)$ \\
\hline$>6$ months & $86(46.0)$ & $43(44.3)$ \\
\hline \multicolumn{3}{|l|}{ BCVA } \\
\hline BCVA (ETDRS letters), mean (SD) in the studied eye & $60.2(14.3)$ & $59.7(15.7)$ \\
\hline \multicolumn{3}{|l|}{ BCVA (ETDRS letters), categories in the studied eye } \\
\hline$<20$ & $3(1.6)$ & $3(3.1)$ \\
\hline $20-59$ & $50(26.7)$ & $25(25.8)$ \\
\hline $60-69$ & $77(41.2)$ & $41(42.3)$ \\
\hline$\geq 70$ & $57(30.5)$ & $28(28.9)$ \\
\hline \multicolumn{3}{|c|}{ Previous ocular treatment for decreased VA due to DME in the studied eye, $n(\%)$} \\
\hline Macular laser & $17(9.1)$ & $9(0.9)$ \\
\hline Other anti-VEGF & $6(3.2)$ & $2(2.0)$ \\
\hline Intravitreal corticosteroids & $8(4.2)$ & $2(2.0)$ \\
\hline Vitrectomy & $3(1.6)$ & $1(1.0)$ \\
\hline \multicolumn{3}{|l|}{ Ocular characteristics in the studied eye } \\
\hline CSFT, $\mu \mathrm{m}$, mean (SD) & $458(142)$ & $411(111)$ \\
\hline IOP, mm Hg, mean (SD) & $15.4(3.4)$ & $15.8(3.5)$ \\
\hline \multicolumn{3}{|l|}{ Comorbidities, $n(\%)$} \\
\hline Arterial hypertension & $154(82.4)$ & $76(78.4)$ \\
\hline Previous cardiovascular disease & $46(24.6)$ & $22(22.7)$ \\
\hline Migraine & $8(4.3)$ & $3(3.1)$ \\
\hline Previous stroke & $12(6.4)$ & $8(8.2)$ \\
\hline Hypercholesterolemia & $75(40.1)$ & $33(34.0)$ \\
\hline Hyperlipidemia & $32(17.1)$ & $15(15.5)$ \\
\hline Sleep disordered breathing & $14(7.5)$ & $8(8.2)$ \\
\hline Diabetic nephropathies & $35(18.7)$ & $19(19.6)$ \\
\hline Diabetic neuropathies & $37(19.8)$ & $16(16.5)$ \\
\hline
\end{tabular}

Percentages are calculated based on the number of patients with available data for that variable. BCVA, bestcorrected visual acuity; BMI, body mass index; CSFT, central subfield thickness; DME, diabetic macular edema; ETDRS, Early Treatment Diabetic Retinopathy Study; HbA1c, glycated hemoglobin; IOP, intraocular pressure; VEGF, vascular endothelial growth factor. 
Table 2. Frequency of ophthalmologist visits and anti-VEGF injections per year over the 36-month study period

Entire cohort Ranibizumab(any injection) only subgroup

\begin{tabular}{lrr}
\hline Year 1 & & \\
$N$ & 242 & 192 \\
Visits, mean (SD) & $13.4(5.1)$ & $12.6(5.0)$ \\
Injections, mean (SD) & $5.1(2.3)$ & $5.1(2.3)$ \\
\hline
\end{tabular}

\begin{tabular}{lcc}
\hline Year 2 & \\
$N$ & 225 & 138 \\
Visits, mean (SD) & $8.8(4.9)$ & $7.3(5.0)$ \\
Injections, mean (SD) & $1.4(2.1)$ & $1.6(2.3)$ \\
\hline
\end{tabular}

\begin{tabular}{lrr}
\hline Year 3 & & \\
$N$ & 187 & 97 \\
Visits, mean (SD) & $8.2(4.9)$ & $6.4(4.5)$ \\
Injections, mean (SD) & $1(2.0)$ & $1.4(2.4)$
\end{tabular}

Total (since inclusion)

Visits, mean (SD) $\quad 30.9(12.2) \quad 26.4(11.4)$

Injections, mean (SD) $\quad 7.6(5.2) \quad 8.0(5.8)$

VEGF, vascular endothelial growth factor; SD, standard deviation.

agnosis of visual impairment due to DME in the studied eye was 9.9 (20.8) months. Mean BCVA at inclusion in the studied eye was 60.2 (14.3) letters. Most patients (85.6\%) had no previous ocular treatment in the studied eye for visual impairment due to DME. Demographics and disease characteristics for the "ranibizumab-only subgroup" were broadly comparable to the overall population (Table 1).

\section{Treatment}

Injections and Visits

Over the 36 months of follow-up, patients in the entire cohort paid their ophthalmologists a mean of 30.9 (12.2) visits and had a mean of 7.6 (5.2) any injections (26.4 visits and 8.0 injections in the subgroup of patients treated only with ranibizumab). The mean ophthalmologist visits and injections was highest in year 1 and decreased in years 2 and 3 (Table 2) for both the entire cohort and the "ranibizumab-only subgroup."

In total, $84.0 \%$ of patients had their first 3 injections within the first 3 months, with a mean of 30.9 (5.4) days between injections. The predominant reason for retreatment at month 3 was systematic injection (corresponding to induction). From month 12 onwards, the main reason

Ranibizumab for DME Visual

Impairment (36 Months, Real World) for retreatment was recurring, persistent, or worsening DME (Table 3).

Treatment Interruptions and Switches

At M36, 178 studied eyes (95.2\%) of the cohort had at least one ranibizumab interruption in the injection retreatment (according to the ophthalmologist). The main reasons for the first interruption were treatment success (95 eyes [53.4\%], defined as maximal BCVA achieved and resolution or regression of DME), treatment failure (44 eyes [24.7\%], defined as no improvement in BCVA and persistence/aggravation of DME), or AEs (9 eyes [5.1\%]).

Of the 187 patients evaluable at M36, another intravitreal treatment was administered after ranibizumab interruption for 90 patients (48.1\%). Patients switched to dexamethasone implant $(n=50[26.7 \%])$, aflibercept $(n=$ 39 [20.9\%]), and ranibizumab/bevacizumab (1 patient). After the first change of treatment, 18 patients (9.6\%) received ranibizumab again. Once patients switched from ranibizumab, they appeared to switch treatments many times thereafter, with no clear patterns observed (Fig. 2).

\section{Visual Acuity}

In the entire cohort, BCVA increased rapidly in the 3 months following ranibizumab initiation, plateaued at month 12, and decreased slightly at M36 (Fig. 3). The BCVA gain at month 12 (primary endpoint) was +7.4 (17.1) letters as previously reported [8]. At M36, mean BCVA was 64.2 (20.1) letters, representing a gain of +4.1 (19.9) letters from baseline. Sensitivity analyses yielded similar BCVA gains at M36 when missing data were imputed using the LOCF method (+4.3 letters) and multiple imputation (+4.0 letters).

The percentage of studied eyes with BCVA $>70$ letters increased from 14.1 at baseline to $45.5 \%$ at month 3 and remained relatively constant through to M36 (42.1\%). At M36, more patients had gains of $\geq 10$ letters (43.9\%) than losses of $\geq 10$ letters $(17.0 \%)$ or stable VA ( -4 to +4 letters; $20.1 \%)$.

BCVA in Patients Treated with Ranibizumab Only

In the "ranibizumab-only subgroup," BCVA did not plateau after month 12, but rather continued to improve up to M36 (Fig. 3). At M36, the visual gain was +10.2 (16.8) letters compared to a gain of +4.1 (19.9) letters in the overall population. In this subgroup, the $62.3 \%$ of the studied eyes had BCVA $>70$ letters at M36 compared to $42.1 \%$ in the overall population. At M36, more patients had gains of $\geq 10$ letters (59.7\%) than losses of $\geq 10$ letters $(11.7 \%)$ or stable VA ( -4 to +4 letters; $13.0 \%)$. The per- 
Table 3. Reasons for ranibizumab retreatment over the 36-month study period

\begin{tabular}{|c|c|c|c|c|c|}
\hline Reason for ranibizumab injection, ${ }^{\mathrm{a}} n(\%)$ & $\begin{array}{l}3 \text { months, }(n=813) \\
\text { injections }\end{array}$ & $\begin{array}{l}12 \text { months, }(n=139) \\
\text { injections }\end{array}$ & $\begin{array}{l}24 \text { months, }(n=146) \\
\text { injections }\end{array}$ & $\begin{array}{l}30 \text { months, }(n=109) \\
\text { injections }\end{array}$ & $\begin{array}{l}36 \text { months, }(n=77) \\
\text { injections }\end{array}$ \\
\hline No data & $2(0.2)$ & $0(0.0)$ & $0(0.0)$ & $1(0.9)$ & $0(0.0)$ \\
\hline Newly decreased BCVA & $3(0.4)$ & $16(11.5)$ & $20(13.7)$ & $18(16.5)$ & $9(11.7)$ \\
\hline Recurrence or worsening of DME & $1(0.1)$ & $29(20.9)$ & $51(34.9)$ & $36(33.0)$ & $23(29.9)$ \\
\hline Persistent DME & $15(1.8)$ & $16(11.5)$ & $31(21.2)$ & $28(25.7)$ & $24(31.2)$ \\
\hline Systematic injection $^{a}$ & $537(66.1)$ & $22(15.8)$ & $13(8.9)$ & $16(14.7)$ & $16(20.8)$ \\
\hline Others & $0(0.0)$ & $0(0.0)$ & $2(1.4)$ & $0(0.0)$ & $0(0.0)$ \\
\hline
\end{tabular}

BCVA, best-corrected visual acuity; DME, diabetic macular edema. ${ }^{a}$ Injections due to baseline, induction, treat and extend, and systematic prevention.

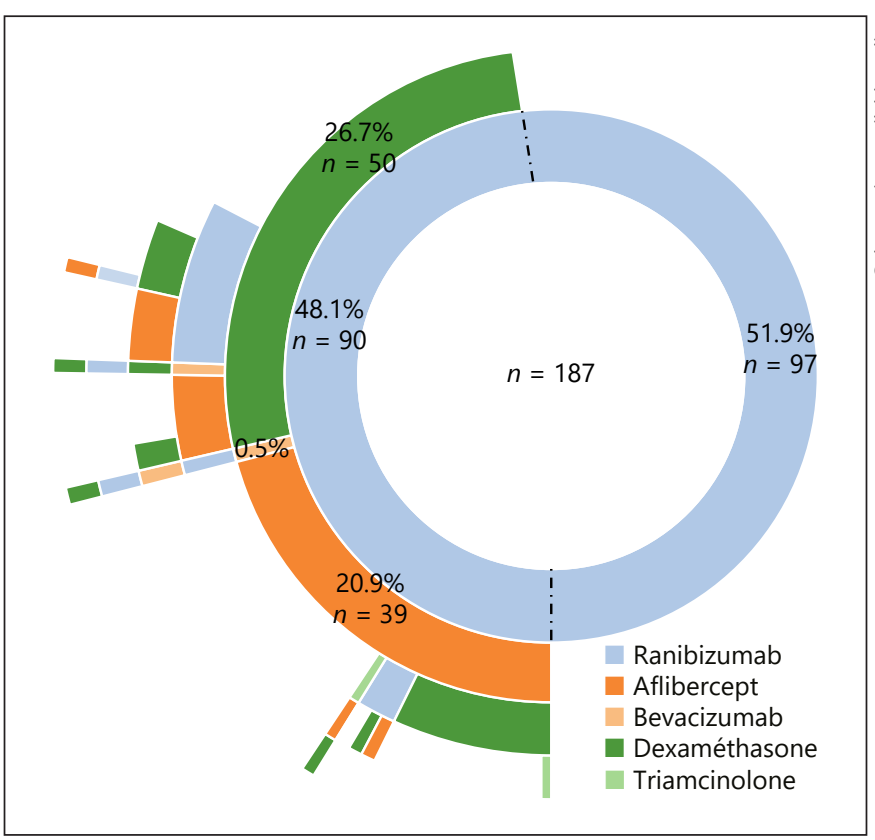

Fig. 2. Intravitreal treatments for diabetic macula edema over the 36-month study.

centage of studied eyes with BCVA $>70$ letters increased from 14.1 at baseline to $45.5 \%$ at month 3 and improved up to M36 to $62.3 \%$.

\section{Other Endpoints}

CSFT improved from month 3 and by M36 had decreased by -127 (138) $\mu \mathrm{m}$ compared to baseline, reaching a mean level of 335 (119) $\mu \mathrm{m}$. Patients in the "ranibizumab-only subgroup" had a CSFT decrease from baseline of
$-116(125) \mu \mathrm{m}$, reaching a mean of $302(95) \mu \mathrm{m}$ at M36 (Fig. 3).

Quality of life questionnaires (NEI-VFQ25 and HUI3) were administered by telephone to $102(54.5 \%)$ of the 187 patients followed at M36 (of whom 94 also had a baseline call). Scores for both questionnaires remained stable throughout the study. Multivariate analysis on the patients followed at M36 and with BCVA data $(n=145)$ found that male gender and milder baseline DME characteristics (BCVA $\geq 59$ and CSFT $<500 \mu \mathrm{m}$ ) were predictive factors for achieving a BCVA of $\geq 70$ letters at M36.

\section{Safety}

Safety was assessed for all patients included (who received at least 1 dose of ranibizumab; $n=290$ ). Thirty patients died throughout the study (see Fig. 1). Over the 36 months, $60.7 \%$ of patients had at least $1 \mathrm{AE}$, and 10 patients had a total of 11 SAEs related to ranibizumab: 2 SAEs of acute hypertensive crisis and 1 SAE each of hypertension, macular perforation, posterior reversible encephalopathy syndrome, myocardial infarction, epistaxis, coronary artery occlusion, ureterolithiasis, vitreal hemorrhage, and retinal vascular disorder. This study did not find any new safety signals, compared to the known profile of ranibizumab.

\section{Discussion}

BOREAL-DME is the first prospective real-life study to follow anti-VEGF treatment of DME patients over a duration of 3 years. Patients in BOREAL demonstrated a BCVA gain of +4.1 letters after 36 months of follow-up 

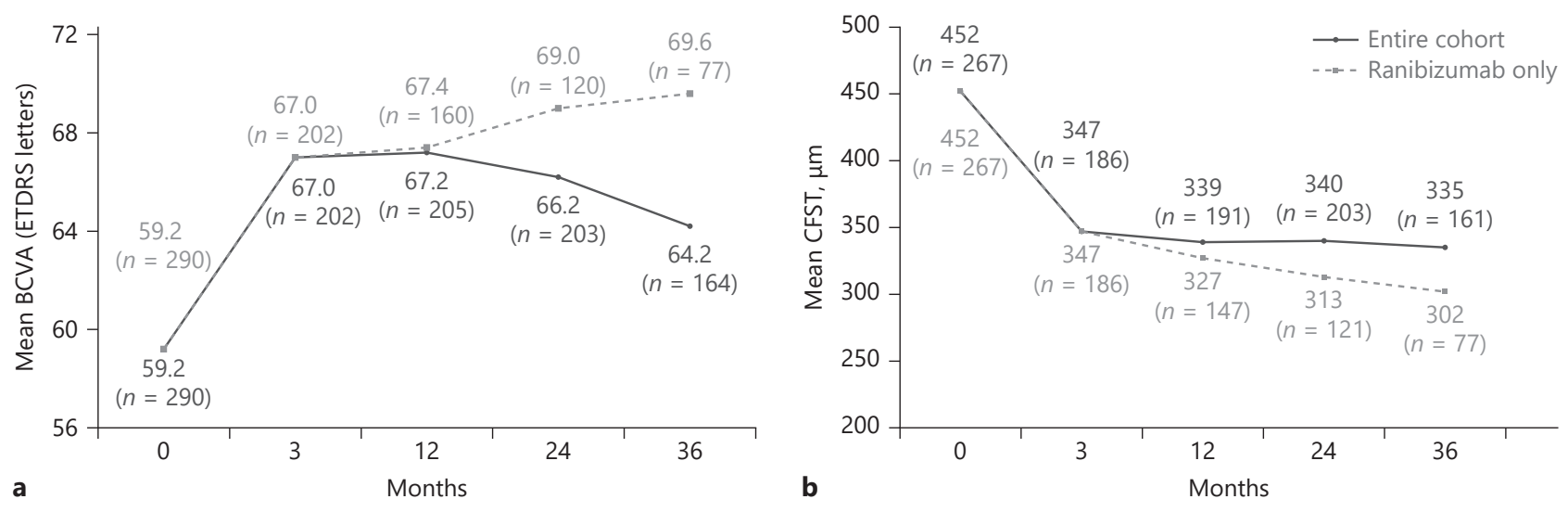

$n=$ number of patients with available data at the follow-up visit

Fig. 3. Mean values for best corrected visual acuity (a) and central subfield thickness (b) over the 36-month study. BCVA, best-corrected visual acuity; CSFT, central subfield thickness.

and a mean reduction from baseline in CSFT of 127 (138) $\mu \mathrm{m}$.

BOREAL followed DME patients from the moment of ranibizumab initiation. This, together with the extended follow-up, allowed us to study not only ranibizumab efficacy in a real-world setting, but also how participating ophthalmologists treat DME and the evolution of the treatment strategy over an extended period.

In BOREAL, BCVA increased rapidly in the 3 months following ranibizumab initiation, then plateaued at month 12 , and decreased slightly at M36. The overall gain in visual acuity from baseline to M36 was +4.1 (19.9) letters in the cohort. These results were supported by similar results in sensitivity analyses with imputation of missing data. This suggests that patients not followed at M36 had similar BCVA values at their last visit compared to the group of patients who had BCVA values at M36.

These gains were lower than those observed at 36 months in the RCTs RESTORE (+8 letters), RISE $(+11$ letters), RIDE (+11.4 letters), and Protocol I (+10 letters in the group ranibizumab with deferred laser). However, in the subgroup of BOREAL patients who were treated exclusively with ranibizumab up to M36, BCVA continued to improve throughout the study, with a final gain at M36 of +10.2 (16.8) letters, notably higher than in the overall population, and in line with RESTORE and other RCTs. This confirms the effectiveness of ranibizumab in a real-life setting but demonstrates that adherence to treatment is required to achieve maximal VA gains. In addition, this subgroup of patients probably represents good responders to ranibizumab and may not reflect how the overall population would respond to continued ranibizumab treatment over 36 months. Indeed, this was despite the fact that the mean number of injections in BOREAL was substantially lower than in the RCTs, especially after the first year. On average, BOREAL patients had 7.6 injections over the 36-month study (8 injections in the subgroup of patients treated only with ranibizum$a b)$, reflecting general undertreatment. By comparison, over 36 months of RCT treatment, RESTORE extension patients had an average of 14.2 injections. The low number of injections in BOREAL was not due to lack of follow-up, with a mean of 30 visits to the ophthalmologist over the 36-month study period. A clinical trial comparing monthly injections to a T\&E regimen found no difference in BCVA after 24 months; however, the median number of injections remained high in both the monthly (22.5 injections) and T\&E (18.5 injections) arms [9].

When BOREAL was initiated, in 2013, treatment recommendations were to start with 3 monthly injections followed by a T\&E or PRN strategy. However since then, treatment recommendations have evolved and now recommend a more intensive strategy in the first year, with at least 7-9 injections [10]. To support these recommendations, a retrospective study has shown that VA gain and VA after 12 months of ranibizumab treatment are sig- 
nificantly higher in patients who received $\geq 7$ injections compared to those who received $<7$ injections [11], suggesting that intensive treatment in the first year is important.

In BOREAL, only $84 \%$ of patients received their first 3 ranibizumab injections within the first 3 months, suggesting that treatment is not always initiated correctly. BOREAL did not record whether patients were treated under a PRN or T\&E regimen. This would have provided us with more information regarding the low number of injections observed in the study. Interestingly, a recent retrospective monocentric study in DME patients with no prior intravitreal pharmacotherapy showed that about a third of eyes with DME responded well with excellent visual and anatomical outcomes using the PRN treatment strategy from the first month without 3 loading doses of ranibizumab. These eyes require fewer injections and follow-up visits compared with those who require 3 loading doses [12].

The longest real-life study in the literature is by Epstein and Amren [13], but is limited by its retrospective monocentric study design. Interestingly, during the 4 years of follow-up, Epstein and colleagues [13] report very similar numbers of injections to our BOREAL study $(4.7,1.4,0.7$, and 0.9 during years $1-4$, respectively) with a slightly lower VA gain at 4 years of +6.2 letters. In a large study $(1,226$ patients among which 774 were treatment naïve), prospective, visual acuity improved at month $12(+4.0$ letters $)$ and at the end of year 2, reaching +5.2 letters at month 24 , similarly to our overall cohort, with also a similar low rate of injections (4.4 during first year and 5.5 over the period study of 2 years) [14]. There have been other previous reports of low injection numbers in real-life studies of ranibizumab in DME $[15,16]$.

With 3 years of follow-up, we believe that BOREAL is the longest prospective real-world study of ranibizumab in DME to date. In BOREAL, the subgroup of patients who stayed on ranibizumab up to M36 had similar or better VA improvements than seen in shorter prospective real-world studies of ranibizumab in DME [1-6]. This subgroup of patients probably represents the good responders to ranibizumab.

By M36, 35.5\% of patients were no longer being followed because they were lost to follow-up or had died. The reasons for dropout do not suggest that these patients dropped out because they did poorly. Subgroup analysis of the demographics and baseline disease characteristics of these patients showed that they were slightly older with more frequent medical history of heart attack and stroke. A similar dropout rate was reported in another prospec- tive observational study of anti-VEGF therapy in DME (39.8\% of patients had dropped out by M24). This study also reported similar VA gains of +4 letters at M12 and +5.2 letters at M24 [14]. Dropout rates in several retrospective observational studies were also high, although methodological differences prevent direct comparisons. Again, VA gains reported in these studies are similar to those observed in BOREAL $[11,13]$. Interestingly, Weiss and colleagues [17] showed that the presence of comorbidities was the most common reason that DME patients stopped treatment.

Diabetes patients have high levels of morbidities in general, and it has previously been shown that DME patients have lower adherence to ranibizumab than patients with neovascular age-related macular degeneration [11]. In the prospective BOREAL study, we observed a high rate of ranibizumab interruption (95.2\%), and almost half of patients (48\%) followed at M36 had switched to other intravitreal treatments, with no clear patterns observed thereafter. Due to the noninterventional study design, we did not collect information about the clinical reasons for switching treatments. This phenomenon of switching is not well documented, in part since most previous observational studies have been retrospective in design. There may be a tendency to switch treatments too quickly, especially given emerging evidence that higher intensity of ranibizumab treatment in year 1 would result in better final visual acuity [11].

We found predictive factors for achieving a BCVA of $\geq 70$ letters at M36 to be male gender and milder baseline DME (BCVA $\geq 59$ and CRT $<500 \mu \mathrm{m}$ ). Response to ranibizumab has previously been shown to be associated with better baseline BCVA, in a retrospective study of 73 DME patients [18]. Post hoc analysis of clinical trial data has also found better baseline BCVA and male gender to predict better visual outcomes [19].

\section{Limitations}

Real-world observational studies, such as BOREAL, impose several constraints on the study design, which can be viewed as limitations. These limitations are discussed in depth in the article describing BOREAL's 12-month results [8]. Briefly, bias could exist due to the self-selection of participating investigators. The potential for bias in the patient population was minimized by the broad inclusion criteria and the fact that ophthalmologists were asked to recruit consecutive patients. Also, while BORE$\mathrm{AL}$ gives valuable insight into real-world treatment practices in France, the results and insights may not be applicable to other countries due to differences in treatment 
recommendations, regulatory approvals, and reimbursement.

The noninterventional study design could not mandate how visual acuity should be measured. Therefore, different methods were used, including ETDRS, Snellen, and Monoyer scales. All visual acuity data were converted into ETDRS letters in order to aggregate data from different sites. This approach is often used in multicenter reallife studies of anti-VEGF therapy [16, 20-22]. The risk of inaccurate results following conversion was further mitigated in BOREAL by the fact that each patient was assessed with the same method throughout the study.

Another limitation in BOREAL was compliance. Of the 290 patients included, $52(26.2 \%)$ were lost to followup by M36, despite the best efforts of investigating ophthalmologists to contact the patients. A similar proportion of patients $(25.45 \%)$ were lost to follow-up in a previous real-world study in DME [11], as mentioned above.

\section{Conclusion}

The 3-year results of BOREAL showed visual results lower than those observed in clinical trials of ranibizum$\mathrm{ab}$, mainly due to undertreatment. Sustained visual and anatomic gains were observed in the subgroup of patients who stayed on ranibizumab throughout the study, representing good responders to treatment. Safety analysis of ranibizumab did not yield any new safety concerns.

\section{Acknowledgements}

The authors thank all the study investigators and the Bordeaux PharmacoEpi (BPE) team who conducted the study. Paid medical writing assistance was provided by Dr. Fiona Dunlevy and Matrix Consultants.

\section{Statement of Ethics}

As stated in the Methods section, all patients signed an informed consent form. The study protocol was approved by the relevant committees in France for data protection (CNIL) and for managing health research data (CCTIRS). In line with French regulations for observational studies, ethical approval was not required.

\section{Conflict of Interest Statement}

P. Massin has received consultancy fees from Novartis, Bayer, Allergan, Horus, and Thea. L. Kodjikian has received consultancy fees from Allergan, Horus, Alimera, Bayer, Novartis, and Thea. C. Creuzot-Garcher has received consultancy fees from Allergan, Alcon, Bayer, Novartis, Roche, Théa, Bausch, and Lomb. A. Glacet Bernard has received consultancy fees from Novartis and Bayer. P.J. Guillausseau has received consultancy fees from Genévrier, Novartis, and Servier. F. Fajnkuchen has received consultancy fees from Allergan, Novartis, and Bayer. J.F. Girmens has received consultancy fees from Allergan, Bayer, and Novartis. C. Delcourt has received consultancy fees from Allergan, Chauvin-Bausch\&Lomb, Théa, and Novartis. P. Blin and A. Grelaud are employees of the BPE Platform that received financial support from Novartis Pharma SAS. F. Guthux is an employee of Novartis Pharma.

\section{Funding Sources}

This study was funded and sponsored by Novartis Pharma SAS, Rueil-Malmaison, France. The author Françoise Guthux is an employee of Novartis and actively participated in the preparation of the manuscript.

\section{Author Contributions}

All authors contributed to the conception and implementation of the study, to the analysis of the results, and to the revision of the manuscript. P. Blin and A. Grelaud designed the computational framework and performed the statistical analysis. P. Massin and F. Guthux wrote the manuscript with input of all other authors.

\section{References}

1 Klein R, Lee KE, Gangnon RE, Klein BE. The 25 -year incidence of visual impairment in type 1 diabetes mellitus: the Wisconsin Epidemiologic Study of Diabetic Retinopathy. Ophthalmology. 2010;117(1):63-70.

2 Moss SE, Klein R, Klein BE. Ten-year incidence of visual loss in a diabetic population. Ophthalmology. 1994;101(6):1061-70.

3 Yau JW, Rogers SL, Kawasaki R, Lamoureux EL, Kowalski JW, Bek T, et al. Global prevalence and major risk factors of diabetic retinopathy. Diabetes Care. 2012;35(3):556-64.
4 Massin P, Bandello F, Garweg JG, Hansen LL, Harding SP, Larsen M, et al. Safety and efficacy of ranibizumab in diabetic macular edema (RESOLVE Study): a 12-month, randomized, controlled, double-masked, multicenter phase II study. Diabetes Care. 2010;33(11): 2399-405.

5 Nguyen QD, Shah SM, Khwaja AA, Channa R, Hatef E, Do DV, et al. Two-year outcomes of the ranibizumab for edema of the mAcula in diabetes (READ-2) study. Ophthalmology. 2010;117(11):2146-51.
6 Mitchell P, Bandello F, Schmidt-Erfurth U, Lang GE, Massin P, Schlingemann RO, et al. The RESTORE study: ranibizumab monotherapy or combined with laser versus laser monotherapy for diabetic macular edema. Ophthalmology. 2011;118(4):615-25.

7 Nguyen QD, Brown DM, Marcus DM, Boyer DS, Patel S, Feiner L, et al. Ranibizumab for diabetic macular edema: results from 2 phase III randomized trials: RISE and RIDE. Ophthalmology. 2012;119(4):789-801.
Ranibizumab for DME Visual Impairment (36 Months, Real World) 
8 Massin P, Creuzot-Garcher C, Kodjikian L, Girmens JF, Delcourt C, Fajnkuchen F, et al. Real-world outcomes with ranibizumab 0.5 $\mathrm{mg}$ in patients with visual impairment due to diabetic macular edema: 12-month results from the 36-month BOREAL-DME study. Ophthalmic Res. 2019;62(2):101-10.

9 Eichenbaum DA, Duerr E, Patel HR, Pollack SM. Monthly versus treat-and-extend ranibizumab for diabetic macular edema: a prospective, randomized trial. Ophthalmic Surg Lasers Imaging Retina. 2018;49(11):e191-7.

10 Creuzot-Garcher C, Massin P. Edèmes maculaires (Société Française d'Ophtalmologie). 2016.

11 Best AL, Fajnkuchen F, Nghiem-Buffet S, Grenet T, Quentel G, Delahaye-Mazza C, et al. Treatment efficacy and compliance in patients with diabetic macular edema treated with ranibizumab in a real-life setting. J Ophthalmol. 2018;2018:4610129.

12 James DGP, Mitkute D, Porter G, Vayalambrone D. Visual outcomes following intravitreal ranibizumab for diabetic macular edema in a pro re nata protocol from baseline: a realworld experience. Asia Pac J Ophthalmol. 2019;8(3):200-5.

13 Epstein D, Amren U. Long-time outcome in patients treated with ranibizumab for diabetic macular edema: a 4-year study. Retina. 2018; 38(1):183-6.
14 Ziemssen F, Wachtlin J, Wachtlin J, Kuehlewein L, Gamulescu M-A, Bertelmann T, et al. Intravitreal ranibizumab therapy for diabetic macular edema in routine practice: two-year real-life data from a non-interventional, multicenter study in Germany. Diabetes Ther. 2018;9(6):2271-89.

15 Egan C, Zhu H, Lee A, Sim D, Mitry D, Bailey C, et al. The United Kingdom Diabetic Retinopathy Electronic Medical Record Users Group, Report 1: baseline characteristics and visual acuity outcomes in eyes treated with intravitreal injections of ranibizumab for diabetic macular oedema. Br J Ophthalmol. 2017;101(1):75-80.

16 Adelman R, Parnes A, Michalewska Z, Parolini B, Boscher C, Ducournau D. Strategy for the management of diabetic macular edema: the European Vitreo-Retinal Society Macular Edema Study. Biomed Res Int. 2015;2015: 352487.

17 Weiss M, Sim DA, Herold T, Schumann RG, Liegl R, Kern C, et al. Compliance and adherence of patients with diabetic macular edema to intravitreal anti-vascular endothelial growth factor therapy in daily practice. Retina. 2018;38(12):2293-300.
18 Chen YP, Wu AL, Chuang CC, Chen SN. Factors influencing clinical outcomes in patients with diabetic macular edema treated with intravitreal ranibizumab: comparison between responder and non-responder cases. Sci Rep. 2019;9(1):10952.

19 Sophie R, Lu N, Campochiaro PA. Predictors of functional and anatomic outcomes in patients with diabetic macular edema treated with ranibizumab. Ophthalmology. 2015; 122(7):1395-401.

20 Shimura M, Kitano S, Muramatsu D, Fukushima $\mathrm{H}$, Takamura Y, Matsumoto $\mathrm{M}$, et al. Real-world management of treatment-naïve diabetic macular oedema in Japan: two-year visual outcomes with and without anti-VEGF therapy in the STREAT-DME study. Br J Ophthalmol. 2020;104(9):1209-15.

21 Stefanickova J, Cunha-Vaz J, Ulbig M, Pearce I, Fernández-Vega Sanz A, Theodossiadis P, et al. A noninterventional study to monitor patients with diabetic macular oedema starting treatment with ranibizumab (POLARIS). Acta Ophthalmol. 2018;96(8):e942-e9.

22 Ziemssen F, Feltgen N, Holz FG, Guthoff R, Ringwald A, Bertelmann T, et al. Demographics of patients receiving Intravitreal antiVEGF treatment in real-world practice: healthcare research data versus randomized controlled trials. BMC Ophthalmol. 2017; 17(1):7. 